\title{
Near field acoustic holography with particle velocity transducers
}

\author{
Jacobsen, Finn; Liu, Yang
}

Published in:

Acoustical Society of America. Journal

Link to article, DOI:

$10.1121 / 1.2082687$

Publication date:

2005

Document Version

Publisher's PDF, also known as Version of record

Link back to DTU Orbit

Citation (APA):

Jacobsen, F., \& Liu, Y. (2005). Near field acoustic holography with particle velocity transducers. Acoustical Society of America. Journal, 118(5), 3139-3144. https://doi.org/10.1121/1.2082687

\section{General rights}

Copyright and moral rights for the publications made accessible in the public portal are retained by the authors and/or other copyright owners and it is a condition of accessing publications that users recognise and abide by the legal requirements associated with these rights.

- Users may download and print one copy of any publication from the public portal for the purpose of private study or research.

- You may not further distribute the material or use it for any profit-making activity or commercial gain

- You may freely distribute the URL identifying the publication in the public portal

If you believe that this document breaches copyright please contact us providing details, and we will remove access to the work immediately and investigate your claim 


\title{
Near field acoustic holography with particle velocity transducers ${ }^{\text {a) }}$
}

\author{
Finn Jacobsen ${ }^{\text {b) }}$ and Yang Liu $^{\text {c) }}$ \\ Acoustic Technology, Ørsted.DTU, Technical University of Denmark, Building 352, Ørsteds Plads, \\ DK-2800 Kgs. Lyngby, Denmark
}

(Received 14 June 2005; revised 26 August 2005; accepted 31 August 2005)

\begin{abstract}
Near field acoustic holography is usually based on measurement of the pressure. This paper describes an investigation of an alternative technique that involves measuring the normal component of the acoustic particle velocity. A simulation study shows that there is no appreciable difference between the quality of predictions of the pressure based on knowledge of the pressure in the measurement plane and predictions of the particle velocity based on knowledge of the particle velocity in the measurement plane. However, when the particle velocity is predicted close to the source on the basis of the pressure measured in a plane further away, high spatial frequency components corresponding to evanescent modes are not only amplified by the distance but also by the wave number ratio $\left(k_{z} / k\right)$. By contrast, when the pressure is predicted close to the source on the basis of the particle velocity measured in a plane further away, high spatial frequency components are reduced by the reciprocal wave number ratio $\left(k / k_{z}\right)$. For the same reason holography based on the particle velocity is less sensitive to transducer mismatch than the conventional technique based on the pressure. These findings are confirmed by an experimental investigation made with a $p-u$ sound intensity probe produced by Microflown. (C) 2005 Acoustical Society of America.
\end{abstract}

[DOI: $10.1121 / 1.2082687]$

PACS number(s): 43.60.Sx, 43.60.Pt, 43.20.Rz [EJS]

Pages: $3139-3144$

\section{INTRODUCTION}

Near field acoustic holography is a powerful experimental technique for analyzing sound fields near sources and for deducing important information about the nature of the sources. ${ }^{1-3}$ In planar near field acoustic holography the sound field is reconstructed from measurements at discrete positions in a finite region in a plane. It is usually the sound pressure that is measured, but in principle there is the same information in the normal component of the acoustic particle velocity. The reason for the fact that conventional near field holography is based on measurements of the sound pressure rather than the particle velocity is of course that it is easier to measure the sound pressure than the particle velocity. However, an acoustic particle velocity transducer called "Microflown" is now available, ${ }^{4,5}$ and therefore it would be interesting to examine the potential of particle velocity-based near field acoustic holography. Thus the purpose of this paper is to compare the performance of particle velocity-based and pressure-based near field holography.

\section{OUTLINE OF THEORY}

Planar near field acoustic holography is based on the fact that the sound pressure in one plane (say, $z=z_{p}>0$ ) can be expressed as the two-dimensional convolution of a "propa-

\footnotetext{
${ }^{a)}$ Portions of this work were presented in "Near field acoustic holography based on an array of particle velocity sensors," Proceedings of Inter-Noise 2005, Rio de Janeiro, Brazil, August 2005.

${ }^{b)}$ Author to whom correspondence should be addressed; electronic mail: fja@oersted.dtu.dk

${ }^{c)}$ Electronic mail: gordan69@163.com
}

gator" and the sound pressure in another plane (say, $z=z_{m}$ $>0$ ), provided that the sources that generate the sound field are confined to half space $(z \leqslant 0)$ and that free-field conditions obtain in the source-free region. The theory was developed in the mid-1980s. ${ }^{1,2}$ Only a brief overview of the most fundamental relations will be presented here; see, e.g., Ref. 3 for more details.

The complex sound pressure $p$ is measured in a plane near the source under examination and a two-dimensional (2D) spatial Fourier transform is calculated. The result is the wave number spectrum,

$$
P\left(k_{x}, k_{y}\right)=\int_{-\infty}^{\infty} \int_{-\infty}^{\infty} p\left(x, y, z_{m}\right) e^{j\left(k_{x} x+k_{y} y\right)} d x d y .
$$

Since the pressure is given by the inverse 2D Fourier transform,

$$
p\left(x, y, z_{m}\right)=\frac{1}{(2 \pi)^{2}} \int_{-\infty}^{\infty} \int_{-\infty}^{\infty} P\left(k_{x}, k_{y}\right) e^{-j\left(k_{x} x+k_{y} y\right)} d k_{x} d k_{y},
$$

it can be seen that the wave number spectrum $P\left(k_{x}, k_{y}\right)$ for any given value of $\left(k_{x}, k_{y}\right)$ inside the radiation circle (that is, when $k_{x}^{2}+k_{y}^{2} \leqslant k^{2}$, where $k=\omega / c$ is the wave number) $)^{3}$ may be interpreted as the amplitude of a plane wave that propagates in the $\left(k_{x}, k_{y}, k_{z}\right)$ direction, where $k_{z}$ must satisfy

$$
k_{x}^{2}+k_{y}^{2}+k_{z}^{2}=k^{2} \text {. }
$$

Outside the radiation circle the wave number spectrum represents the amplitude of an evanescent wave. Thus the wave number transform provides an expansion of the sound field into plane and evanescent waves. It now follows that one can 
determine the wave number spectrum of the sound pressure in another, parallel plane, simply by multiplying the wave number spectrum in the measurement plane with an exponential propagator,

$$
G\left(z_{p}, z_{m}, k_{x}, k_{y}\right)=e^{-j k_{z}\left(z_{p}-z_{m}\right)},
$$

where $k_{z}$ is given by

$$
k_{z}= \begin{cases}\sqrt{k^{2}-k_{x}^{2}-k_{y}^{2}} & \text { for } k_{x}^{2}+k_{y}^{2} \leqslant k^{2} \\ -j \sqrt{k_{x}^{2}+k_{y}^{2}-k^{2}} & \text { for } k_{x}^{2}+k_{y}^{2}>k^{2}\end{cases}
$$

The sign in the last equation has been chosen so as to satisfy the Sommerfeld radiation condition with the $e^{j \omega t}$ sign convention. Eventually one can calculate the sound pressure in the prediction plane by an inverse Fourier transform. One can also calculate the particle velocity vector, and thus the sound intensity. For example, the wave number spectrum of the normal component of the particle velocity in the prediction plane equals the wave number spectrum of the pressure in the measurement plane multiplied with the propagator

$$
G_{p u}\left(z_{p}, z_{m}, k_{x}, k_{y}\right)=\frac{k_{z}}{\rho c k} e^{-j k_{z}\left(z_{p}-z_{m}\right)},
$$

where $\rho c$ is the characteristic impedance of the medium. ${ }^{2,3}$

An alternative technique involves measuring the normal component of the particle velocity in a plane, transform to the wave number domain, multiply with the propagator given by Eq. (4), and transform back to the spatial domain. The result is the normal component of the particle velocity in the prediction plane. If instead the wave number spectrum is multiplied by the propagator

$$
G_{u p}\left(z_{p}, z_{m}, k_{x}, k_{y}\right)=\frac{\rho c k}{k_{z}} e^{-j k_{z}\left(z_{p}-z_{m}\right)}
$$

before the inverse Fourier transform is carried out one will get the sound pressure in the prediction plane. ${ }^{2,3}$

The above-noted description is, of course, grossly oversimplified. In practice the measurement area must obviously be finite, the sound field is sampled only at a finite number of discrete positions, and the 2D spatial Fourier transform is approximated by a $2 \mathrm{D}$ discrete Fourier transform. ${ }^{1-3}$ To reduce the influence of the finite aperture and the spatial sampling, zero padding and a tapered spatial window should be applied before the discrete spatial Fourier transform is calculated. $^{2}$ The spatial sampling must satisfy the sampling theorem, and therefore one cannot sample very close to the source where there may be high spatial frequency components. On the other hand, one should not sample too far away, because then the evanescent modes will be buried in noise. ${ }^{2,3}$ Moreover, whereas forward prediction is numerically stable, backward prediction, which involves predicting the sound field closer to the source than the measurement plane, is an unstable "ill-posed" inverse problem that requires regularization because of the fact that the evanescent waves are amplified exponentially with the distance. The standard regularization technique involves spatial low-pass filtering (multiplying with a window in the wave number domain). See, e.g., Refs. 2 and 3. Finally, it should be men- tioned that the velocity-to-pressure propagator given by Eq. (7) has a singularity on the radiation circle where $k_{z}$ goes to zero.

\section{A SIMULATION STUDY}

A simulation study has been carried out. To obtain realistic results a model of a relatively complicated source, a simply supported vibrating steel plate in an infinite, rigid baffle, was developed. The dimensions of the plate were $0.45 \times 0.75 \mathrm{~m}$, and it was $5 \mathrm{~mm}$ thick, corresponding to a coincidence frequency of about $2.4 \mathrm{kHz}$. The lowest eigenfrequency of the plate was about $80 \mathrm{~Hz}$. The excitation of the plate was a harmonic point source of $10 \mathrm{~N}$ placed near one of the corners $(10 \mathrm{~cm}$ from each edge), and the resulting vibrational velocity was calculated using a conventional modal summation. The vibrating plate was divided into 64 $\times 64$ rectangles, each of which was regarded as a point source on the baffle, and the sound pressure radiated by the plate was calculated from the corresponding approximation to Rayleigh's first integral. ${ }^{3}$ A virtual microphone array of dimensions $1.5 \times 1.5 \mathrm{~m}$ with $32 \times 32$ microphones (corresponding to a sampling distance of $4.7 \mathrm{~cm}$ ) was placed $15 \mathrm{~cm}$ above the plate, and the prediction plane was $5 \mathrm{~cm}$ above the plate.

The spatial window was an 8-point Tukey window, ${ }^{3}$ and zeros were added outside the measurement area to reduce wrap-around errors. The regularization was an exponential $k$-space low-pass window with a cut-off frequency of 0.6 times the Nyquist frequency and a value of 0.1 of the parameter $\alpha{ }^{3}$ To reduce the influence of the singularity of Eq. (7) on the radiation circle the wave number spectra were smoothed as described in Ref. 6.

In what follows, "true" values of quantities in the prediction plane have been calculated directly from the approximated Rayleigh's integral, whereas predictions have been calculated from the "measured" sound pressure or particle velocity. The results are shown along a diagonal line in the prediction plane from the corner nearest the excitation point to the opposite corner.

\section{A. Ideal transducers}

Figure 1(a) shows the "true" sound pressure level at $200 \mathrm{~Hz}$, a prediction based on the pressure in the measurement plane, and a prediction based on the normal component of the particle velocity in the measurement plane. There is good agreement between the "true" pressure and both predictions in a significant part of the prediction plane, but near the edges the agreement deteriorates.

Figure 1(b) shows a similar comparison of the "true" normal component of the particle velocity level and predictions based on the pressure and based on the particle velocity in the measurement plane. (Note that the particle velocity level is shown relative to a reference velocity of $50 \mathrm{~nm} / \mathrm{s}$ so as to make it directly comparable with the sound pressure level.) The two predictions are in good agreement with the "true" value, but it is apparent that the velocity-based prediction is far better than the pressure-based prediction near the edges. Similar results have been found at other frequencies. 

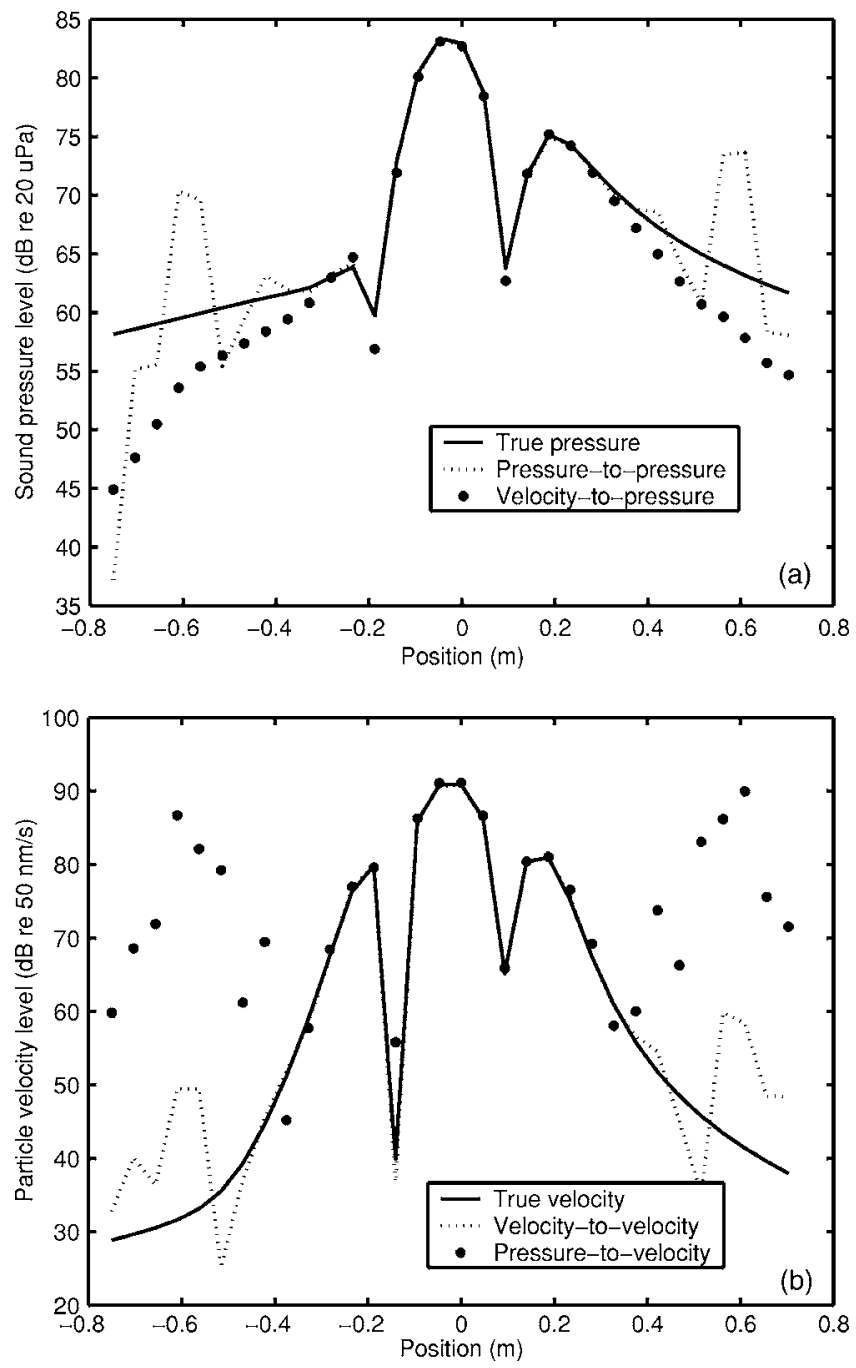

FIG. 1. "True" and predicted sound pressure level (a) and particle velocity level (b) at $200 \mathrm{~Hz}$ along a line across the prediction plane $5 \mathrm{~cm}$ from the vibrating plate.

Note also that the "true" particle velocity, not unexpectedly, decays faster toward the edges and has a larger dynamic range than the pressure. This is favorable for holography based on the particle velocity, since it means that leakage caused by the spatial window is reduced. However, the main reason for the consistent observation that velocity-based predictions of the pressure are considerably better than pressurebased predictions of the velocity is undoubtedly the fact that the wave number factor used in the former case reduces high spatial frequencies whereas the reciprocal factor used in the latter case amplifies them. These effects are demonstrated by Fig. 2, which shows the amplitude of the propagators given by Eqs. (4), (6), and (7) (except for the $\rho c$ factor), corresponding to the predictions shown in Fig. 1. Amplification of high spatial frequencies increases the inherent numerical instability in backward propagation. ${ }^{3}$

Figure 3 shows the "true" normal component of the sound intensity level at $200 \mathrm{~Hz}$ along the diagonal line across the prediction plane, a prediction based on the pressure in the measurement plane, a prediction based on the corresponding normal component of the particle velocity, and a prediction in which the pressure in the expression for

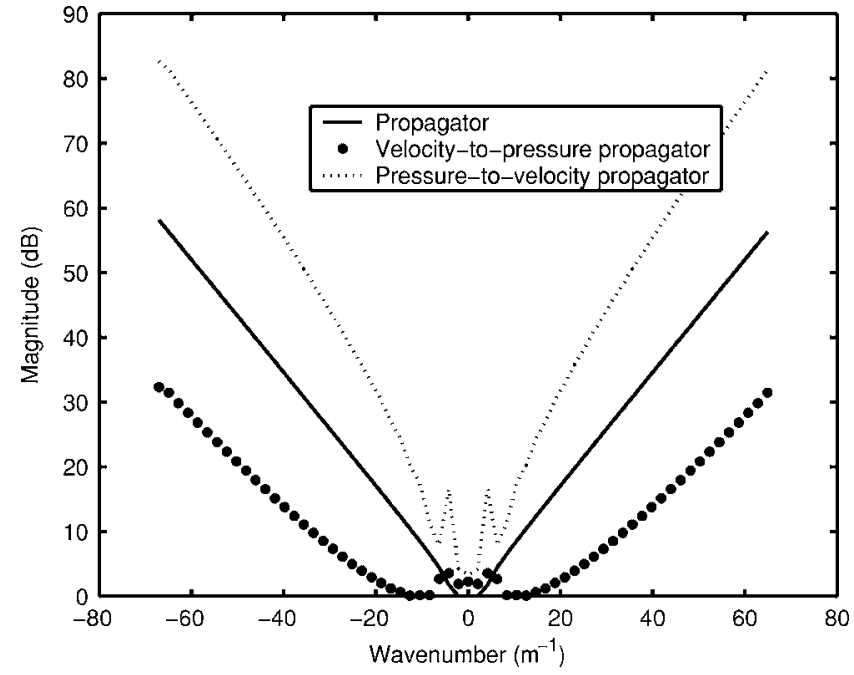

FIG. 2. The three propagators [Eqs. (4), (6), and (7)] used for the predictions shown in Fig. 1.

the intensity has been calculated from the pressure in the measurement plane and the particle velocity in the same expression has been calculated from the particle velocity in the measurement plane. Note the low level compared with the sound pressure level, and note the regions of negative intensity; the panel is a very inefficient radiator of sound at $200 \mathrm{~Hz}$. It seems that the prediction in which the pressure has been based on the pressure and the velocity on the velocity is slightly better than the prediction based solely on the velocity and much better than the prediction based solely on the pressure. Similar observations have been made at other frequencies. The reason for the slightly better performance of the prediction based both on the pressure and the particle velocity compared with the prediction based exclusively on the velocity is perhaps that inaccuracies caused by the smoothing that is needed in velocity-to-pressure predictions [Eq. (7)] are avoided.
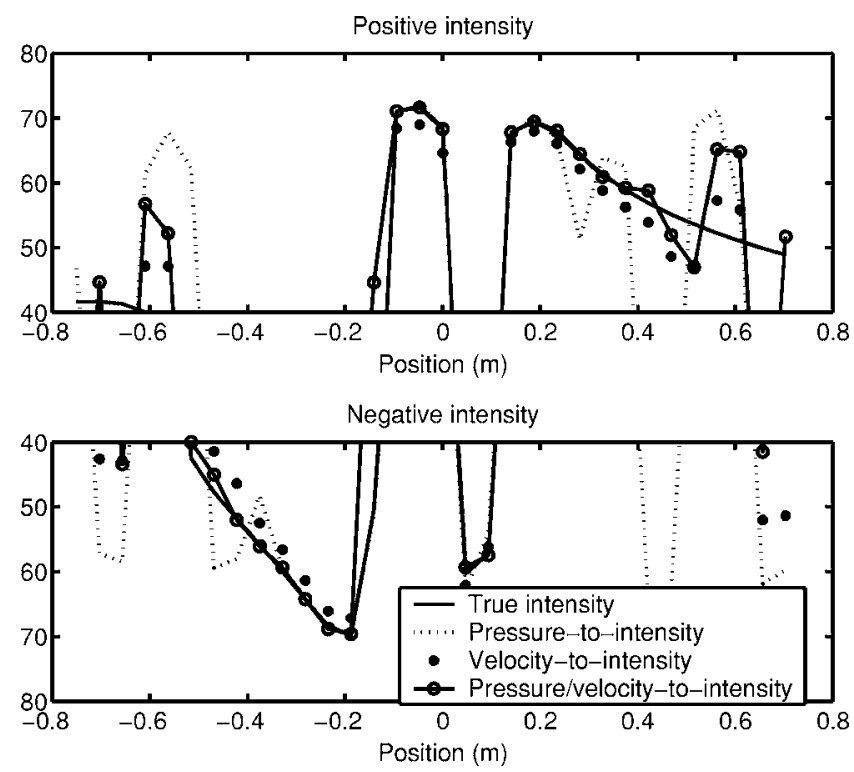

FIG. 3. "True" and predicted sound intensity level at $200 \mathrm{~Hz}$ along a line across the prediction plane $5 \mathrm{~cm}$ from the vibrating plate.

F. Jacobsen and Y. Liu: Holography with velocity transducers 3141 

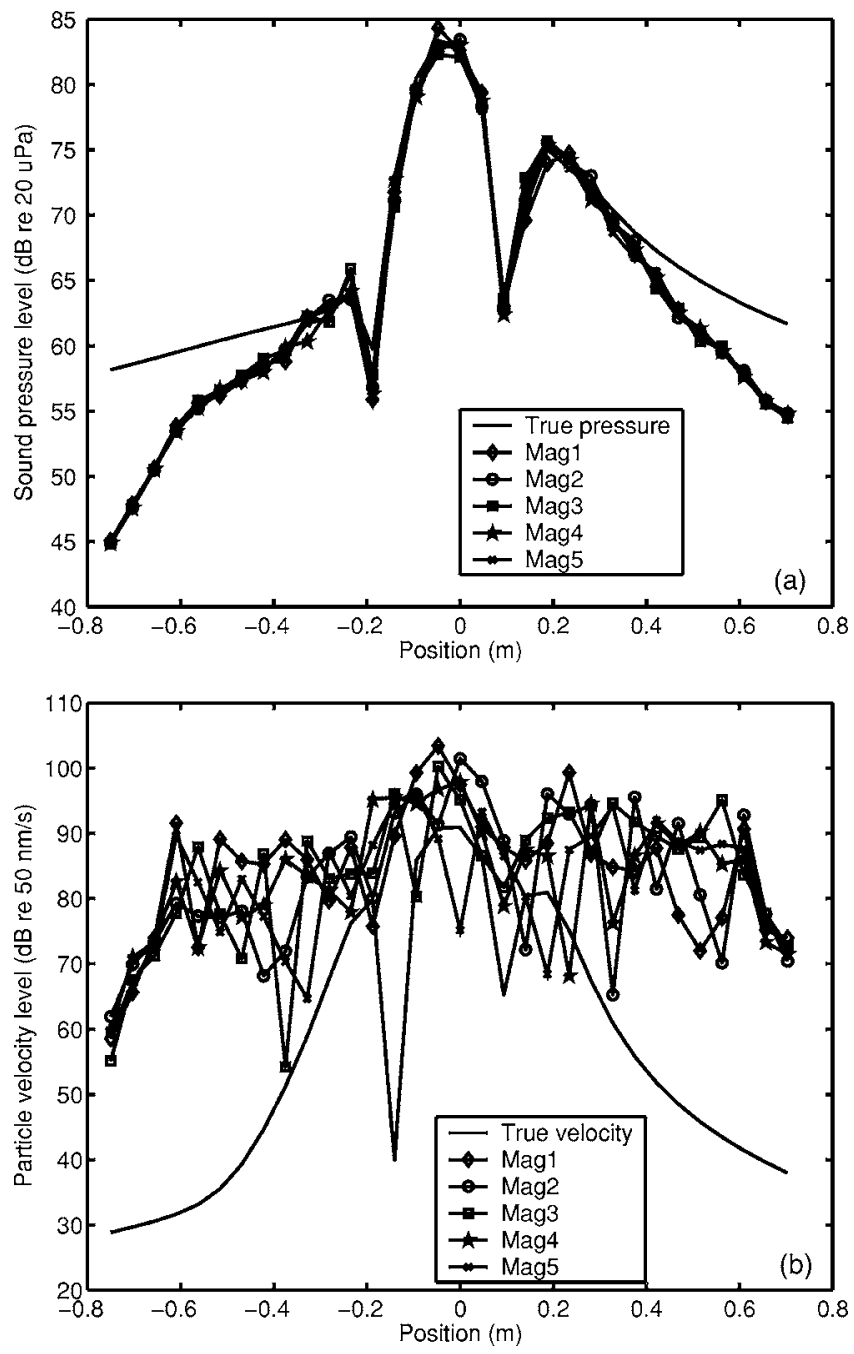

FIG. 4. "True" and predicted sound pressure level (a) and particle velocity level (b) at $200 \mathrm{~Hz}$. The sound pressure level in (a) is predicted from particle velocity "measurements" in which random amplitude mismatch has been introduced, and the particle velocity level in (b) is predicted from pressure "measurements" in which random amplitude mismatch has been introduced.

\section{B. Transducer mismatch}

In stationary sound fields one can measure the pressure or particle velocity in the measurement plane point by point with a single transducer using a reference signal (or several reference signals, depending on the complexity of the source mechanism). ${ }^{7}$ On the other hand, if the sound field is generated by a nonstationary source it is necessary to measure the complete sound field in the measurement plane at the same time, and thus a full two-dimensional transducer array must be used. ${ }^{8}$ However, in real measurements with transducer arrays a certain amount of amplitude and phase mismatch between the transducers can be expected. The effect of such measurement inaccuracies on pressure-to-pressure predictions has been examined by Nam and Kim. ${ }^{9}$ To examine the effect on "cross predictions," that is, predictions where the pressure is calculated from the particle velocity and the particle velocity is calculated from the pressure, random amplitude mismatch, uniformly distributed between -0.5 and $0.5 \mathrm{~dB}$, has been introduced in the above-described simulations. Figure 4 shows the results of five outcomes of this
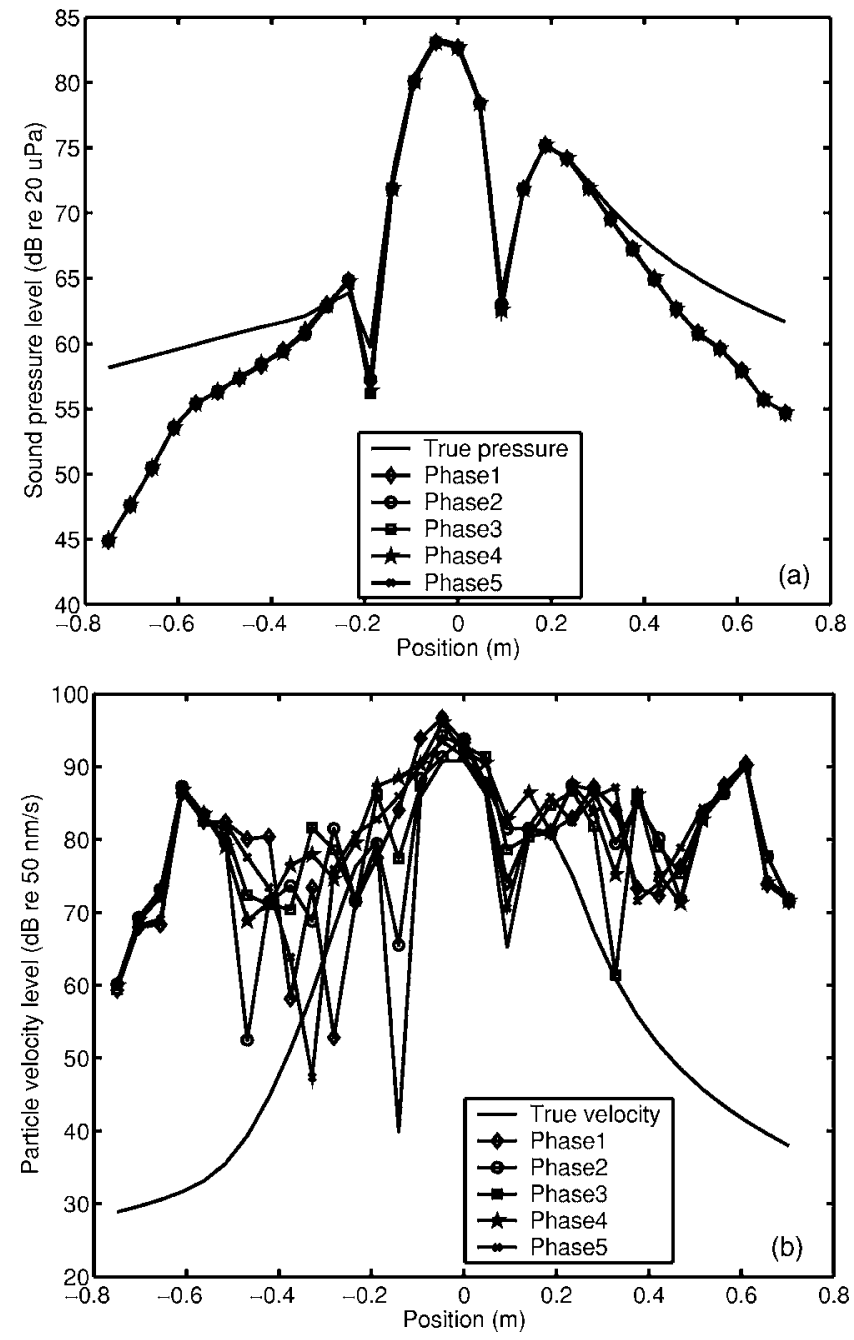

FIG. 5. "True" and predicted sound pressure level (a) and particle velocity level (b) at $200 \mathrm{~Hz}$. The sound pressure level in (a) is predicted from particle velocity "measurements" in which random phase mismatch has been introduced, and the particle velocity level in (b) is predicted from pressure "measurements" in which random phase mismatch has been introduced.

stochastic experiment. It is apparent that whereas the influence on predictions from velocity to pressure is fairly moderated predictions from pressure to velocity are extremely seriously affected. The explanation is that the white noise introduced in the wave number spectrum by these errors is amplified by the wave number ratio $\left(k_{z} / k\right)$ in the pressurebased cross predictions and reduced by the reciprocal factor in the velocity-based cross predictions.

Figure 5 shows the results of similar stochastic experiments in which phase errors uniformly distributed from $-2^{\circ}$ to $2^{\circ}$ have been introduced in the cross predictions. The results resemble the results shown in Fig. 4, but this amount of random phase mismatch appears to be slightly less serious than random amplitude mismatch in the interval from -0.5 to $0.5 \mathrm{~dB}$.

\section{EXPERIMENTAL RESULTS}

To examine the validity of the results of the simulations some experiments have been carried out. The sound source was a $3-\mathrm{mm}$ steel plate with dimensions $39 \times 63 \mathrm{~cm}$ 
mounted in a large baffle. The radiating plate was one of the side plates of a box; and one of the other side plates of the box was driven via a Brüel \& Kjær (B\&K) 8200 force transducer by a B\&K 4809 electrodynamic exciter fed with wideband pink random noise. The signal from the force transducer was passed through a B\&K 2635 charge amplifier and used as a reference signal. The box was suspended from a support in such a way that it did not touch the rectangular hole in the baffle; the resulting leaks around the plate were sealed by tape. The frequency responses between sound pressure and the reference signal and between the normal component of the particle velocity and the reference signal were measured at $18 \times 28$ points in two planes using a Microflown $\frac{1}{2}$-in. $p-u$ sound intensity probe. The sampling distance was $5 \mathrm{~cm}$.

Since the measurements took place in an ordinary room the image source resulting from the reflecting floor had to be taken into account; that is, the sound field was sampled down to half a sampling distance above the floor, and the sound field was considered to be symmetrical about the plane of the floor. $^{7}$ Thus the resulting spatial transform was a $18 \times 56$ point transform. The phase and amplitude calibration of the Microflown particle velocity transducers was carried out in an impedance tube as described in Ref. 10. A robot moved the transducer over the two measurement planes, one typically about $12 \mathrm{~cm}$ from the source plane, and one typically about $4 \mathrm{~cm}$ from the source plane. A B\&K 3560 "Pulse" analyzer in the $1 / 24$ octave mode was used, and the measurements and the robot were controlled by B\&K's program "Spatial transformation of sound fields" (STSF). The data were, however, postprocessed not with STSF but with the same MATLAB routines as used in the simulations. Each set of $1 / 24$ octave data was processed individually and then summed so as to produce the sound pressure, the particle velocity, and the sound intensity in one-third octave bands. (With measurements directly in one-third octave bands the coherence between the force signal and the pressure and particle velocity was too poor; strictly speaking the theory outlined in Sec. II is based on pure-tone excitation, and onethird octave bands are too wide. Otherwise expressed, with one-third octave noise the physical dimensions of the source exceeded the correlation distance. ${ }^{7}$ )

Figure 6 shows an example of the results. Figure 6(a) shows a comparison of the "true," that is, directly measured sound pressure level in the $200-\mathrm{Hz}$ one-third octave band along a vertical line midway through the plane close to the panel and predictions of the same quantity based on measurements of the pressure and the normal component of the particle velocity in the plane further away. Note that the sound pressure and the particle velocity increase toward the reflecting floor (the position at $0 \mathrm{~m}$ ). It can be seen that the velocity-based prediction is acceptable and on the whole somewhat more accurate than the pressure-based prediction.

Figure 6(b) shows the "true" particle velocity in the plane close to the panel and predictions based on measurements of the pressure and the particle velocity in the plane further away. It is clear that the pressure-based prediction tends to overestimate the particle velocity and that the velocity-based prediction is by far the best.
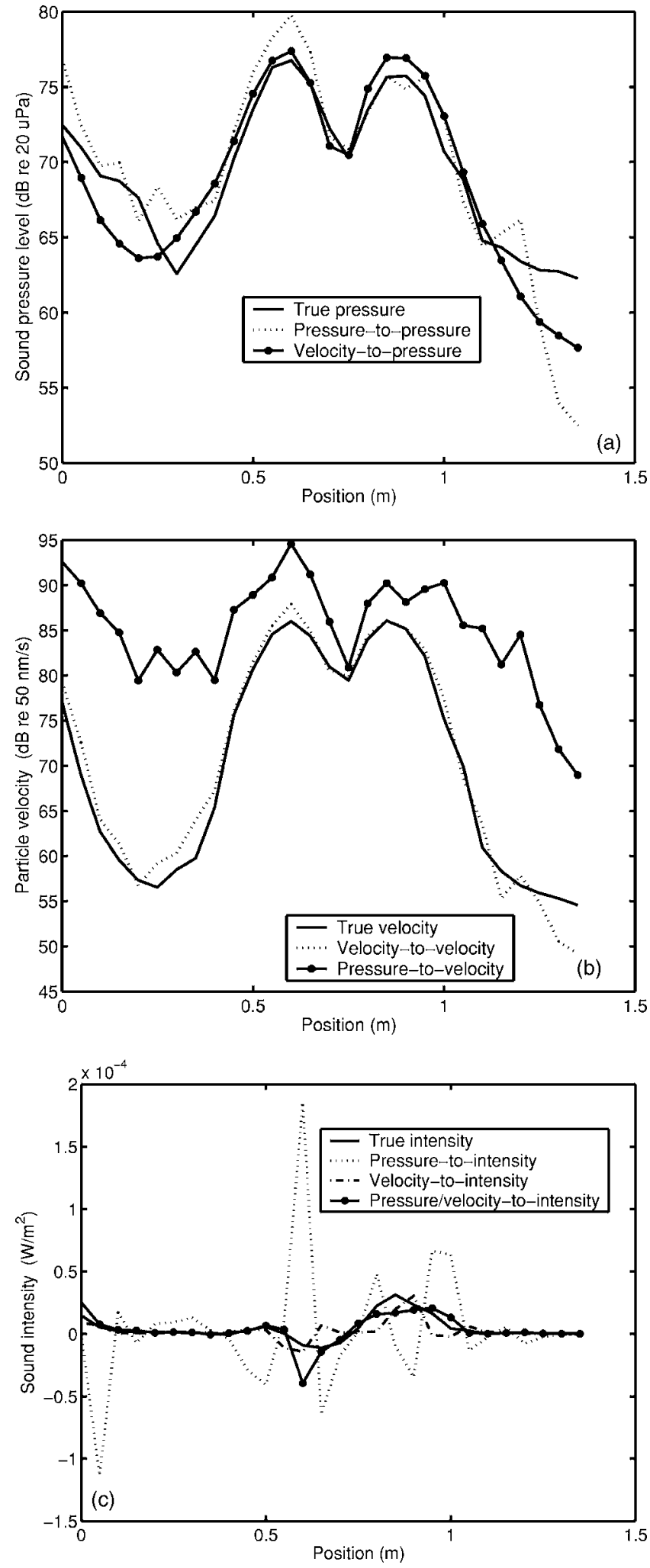

FIG. 6. Measured sound pressure level (a), particle velocity level (b), and sound intensity (c) in the $200-\mathrm{Hz}$ one-third octave band compared with predictions based on the pressure and on the particle velocity in the measurement plane.

Finally Fig. 6(c) shows the "true" sound intensity in the plane close to the panel and a prediction based solely on pressure measurements in the plane further away, a prediction based solely on particle velocity measurements in the 
same measurement plane, and a prediction based on both pressure and particle velocity measurements. Again the velocity-based prediction performs much better than the prediction based on measurements of the pressure, and again the prediction based on measurements of both quantities seems to be slightly better than the prediction based solely on the velocity. Similar results have been found in other one-third octave bands.

\section{CONCLUSIONS}

Planar near field acoustic holography has traditionally been based on measurements of the sound pressure, but since an acoustic particle velocity transducer is now available this paper has examined near field holography based on measurements of the normal component of the particle velocity.

A simulation study has revealed that the particle velocity decays faster toward the edges of the measurement region than the sound pressure and has a larger dynamic range; thus spatial windowing has less serious consequences on velocitybased holography than on conventional pressure-based holography. Nevertheless, it has generally been observed that the quality of pressure-to-pressure predictions is similar to the quality of velocity-to-velocity predictions. However, velocity-to-pressure backward predictions are far better than pressure-to-velocity backward predictions because of the fact that the wave number ratio that enters into such cross predictions reduces high spatial frequencies in the former case but amplifies them in the latter case.

For the same reason amplitude and phase mismatch, which is likely to occur in measurements with arrays of transducers, has a far more serious influence on pressure-tovelocity predictions than on velocity-to-pressure predictions: such transducer mismatch introduces high spatial frequencies, and the resulting errors are amplified exponentially with the distance if the prediction plane is closer to the source than the measurement plane.

The superiority of the method based on measurement of the particle velocity has been confirmed by an experimental study in which the sound pressure and the normal component of the particle velocity were measured at some distance from a vibrating, baffled steel panel with a Microflown $p-u$ sound intensity probe and used to predict the pressure, the normal component of the particle velocity, and the normal component of the sound intensity in a plane closer to the panel. Velocity-based predictions were consistently found to be better than pressure-based predictions. Thus if only one type of transducer is available one should choose to measure the particle velocity. However, slightly better predictions of the sound intensity may be obtained if the pressure that enters into this quantity is predicted from the pressure and the particle velocity is predicted from the particle velocity.

\section{ACKNOWLEDGMENTS}

The authors would like to thank Microflown Technologies for lending us a $p-u$ sound intensity probe. We are also indebted to Brüel \& Kjær for lending us a robot, a multichannel "Pulse" front-end, and license to use the STSF software, and to Jørgen Hald, Brüel \& Kjær, for useful advice during the project.

${ }^{1}$ J. D. Maynard, E. G. Williams, and Y. Lee, "Nearfield acoustic holography. I. Theory of generalized holography and the development of NAH," J. Acoust. Soc. Am. 78, 1395-1413 (1985).

${ }^{2}$ W. A. Veronesi and J. D. Maynard, "Nearfield acoustic holography (NAH). II. Holographic reconstruction algorithms and computer implementation," J. Acoust. Soc. Am. 81, 1307-1322 (1987).

${ }^{3}$ E. G. Williams, Fourier Acoustics—Sound Radiation and Nearfield Acoustical Holography (Academic Press, San Diego, 1999).

${ }^{4}$ R. Raangs, W. F. Druyvesteyn, and H.-E. de Bree, "A low-cost intensity probe," J. Audio Eng. Soc. 51, 344-357 (2003).

${ }^{5}$ H.-E. de Bree, "The Microflown: An acoustic particle velocity sensor," Acoust. Aust. 31, 91-94 (2003).

${ }^{6} \mathrm{E}$. G. Williams and J. D. Maynard, "Numerical evaluation of the Rayleigh integral for planar radiators using the FFT," J. Acoust. Soc. Am. 72, 2020-2030 (1982).

${ }^{7}$ K. B. Ginn and J. Hald, "STSF-Practical instrumentation and applications," Brüel \& Kjær Technical Review 2, 1-27 (1989).

${ }^{8}$ J. Hald, "Non-stationary STSF," Brüel \& Kjær Technical Review 1, 1-36 (2000).

${ }^{9}$ K.-U. Nam and Y.-H. Kim, "Errors due to sensor and position mismatch in planar acoustic holography," J. Acoust. Soc. Am. 106, 1655-1665 (1999).

${ }^{10} \mathrm{~F}$. Jacobsen and H.-E. de Bree, "Measurement of sound intensity: $p-u$ probes versus $p$ - $p$ probes," Proceedings of Noise and Vibration Emerging Methods 2005, Saint Raphaël, France. 